\title{
A Framework for Comprehensive Analysis of a Swing in Sports Using Low-Cost Inertial Sensors
}

\author{
Amin Ahmadi, Francois Destelle, \\ David Monaghan, Noel E. O'Connor \\ Insight Center for Data Analytics \\ Dublin City University \\ Dublin, Ireland
}

\author{
Chris Richter, Kieran Moran \\ Applied Sports Performance Research \\ School of Health and Human Performance \\ Dublin City University \\ Dublin, Ireland
}

\begin{abstract}
We present a novel framework to monitor the threedimensional trajectory (orientation and position) of a golf swing using miniaturized inertial sensors. Firstly we employed a highly accurate and computationally efficient revised gradient descent algorithm to obtain the orientation of a golf club. Secondly, we designed a series of digital filters to determine the backward and forward segments of the swing, enabling us to calculate drift-free linear velocity along with the relative 3D position of the golf club during the entire swing. Finally, the calculated motion trajectory was verified against a ground truth VICON system using Iterative Closest Point (ICP) in conjunction with Principal Component Analysis (PCA). The computationally efficient framework present here achieves a high level of accuracy $(r=0.9885, p<0.0001)$ for such a low-cost system. This framework can be utilized for reliable movement technique evaluation and can provide near real-time feedback for athletes in various unconstrained environments. It is envisaged that the proposed framework is applicable to other racket based sports (e.g. tennis, cricket and hurling).
\end{abstract}

\section{INTRODUCTION}

Success in sport is highly dependent upon the movement technique/motion of the athlete and the instrument they are swinging. In golf for example, the motion of the golf club is the main determining factor in the ball's launch characteristics and ultimately determines the golf ball's trajectory and final position. For sport instruments (e.g. golf club, tennis racket, hurl) the key motion features are 3 dimensional orientation, velocity and displacement. Clearly, in order to provide the athlete with useful technique feedback, it is imperatie that we can accurately determine these measures in near-real time during key phases (e.g. backswing, forward swing). Traditionally, such analyses utilize multiple (6+ typically) camera-based motion analysis systems (e.g. VICON Motion Systems Ltd, Oxford, UK) which require complicated and time consuming setup and calibrations. More importantly, such systems are fixed to a setup location, require time-consuming post processing and can be very expensive. This makes their utilization very problematic and lacking ecological validity because they cannot be easily used in the natural sporting environment.

Improvements in microelectronics and other micro technologies have made it possible to take advantage of using miniaturized, light, inexpensive Micro-Electronic-Mechanical (MEMS) inertial sensors, including accelerometers and gyroscopes to classify sporting activities [1], [2], [3] and analyze athletes' performance in real environments [4], [5]. Over the last decade, a number of methodologies have been proposed to investigate translational and rotational motion of an instrument (or athlete's body segment) using inertial sensors, which circumvent many of the above limitations of camera based systems. Most of these approaches utilize Kalman filters to estimate the orientation. However, utilizing Kalman filters to measure orientation has some disadvantages including implementation complexity, high sampling rate due to linear regression iteration and the requirement to deal with large scale vectors to describe rotational kinematics in threedimensions [6], [7]. Therefore, we opted for employing an algorithm which has been shown to provide effective performance at low computational expense. Using such a technique, it is feasible to have a lightweight, inexpensive system capable of detecting various phases of a complex motion (high speed 3D motion in space) in near real-time, which is important in sport and exercise biomechanics. The objective of this paper is to investigate and report on the accuracy of our system to detect the $3 \mathrm{D}$ position of the club during the backward swing and the forward swing.

\section{PROPOSED FRAMEWORK}

The main components of our framework used to calculate 3D orientation and 3D position of an instrument during a swing are illustrated in Fig.1. As can be seen, signals from a tri-axial accelerometer and gyroscope are required to calculate sensor orientation with respect to the global frame. The calculated orientation along with the series of low pass and high pass digital filters are then employed to yield position.

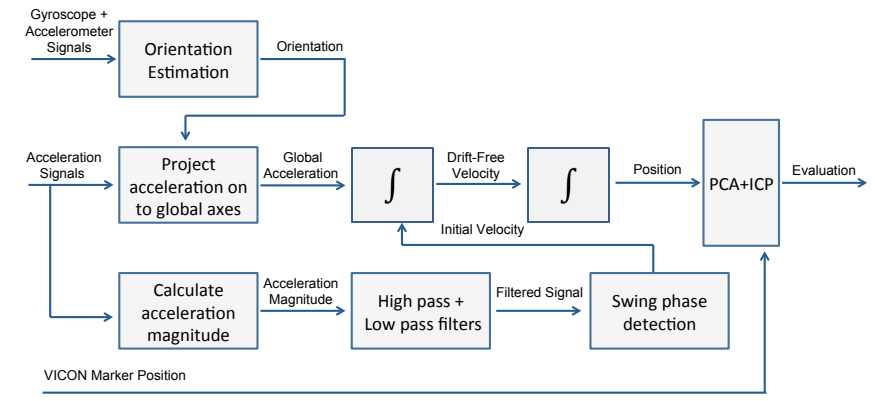

Fig. 1. The main components of the proposed framework

\section{A. Experiment Setup}

We used low-cost inertial sensors as they are gaining in popularity in the area of human and instrument movement 
monitoring and due to their accuracy and potential for real time applications. These sensors are capable of measuring linear acceleration, angular velocity, and gravitational forces and are often used in Motion Capture systems. Technical specifications of the inertial sensors we utilized are summarized in Table I.

TABLE I. TECHNICAL SPECIFICATIONS OF THE INERTIAL SENSOR UNITS.

\begin{tabular}{|l|l|}
\hline Features & Values \\
\hline Resolution (Acc, Gyr, Mag) & 12 bit, 16 bits, 12 bits respectively \\
\hline Sampling rate & Scalable up to $512 \mathrm{~Hz}$ \\
\hline & $\begin{array}{l}\text { Acc: scalable up to } \pm 8 \mathrm{G} \\
\text { Gyro: scalable up to } \pm 2000 / \mathrm{s} \\
\text { Mag: scalable up to } \pm 8.1 \mathrm{G}\end{array}$ \\
\hline Connectivity & Bluetooth-Class $1(100 \mathrm{~m}$ range), Micro SD card \\
\hline Dimension & $57 \times 38 \times 21 \mathrm{~mm}$ \\
\hline Weight & $49 \mathrm{~g}$ including housing and battery \\
\hline
\end{tabular}

We used the VICON optical monitoring system (gold standard) to verify the results obtained from the inertial sensors. Twelve cameras were used to record the the position of refelctive markers placed on the golf club at 250 frames per second. The placement of VICON optical markers and the inertial sensor unit attached on the golf club is illustrated in Fig2. For this study, six different golf swings performed by a novice player were captured using VICON and one inertial sensor device.

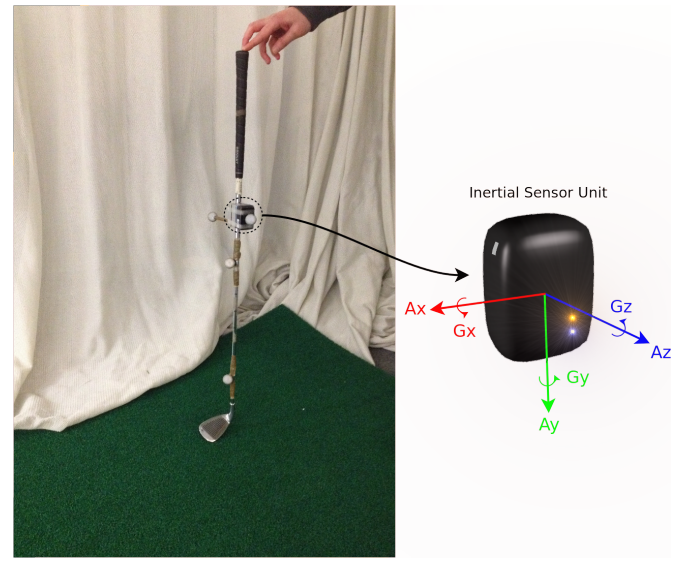

Fig. 2. Placement of VICON optical markers and one inertial sensor unit on a golf club is shown

\section{B. Orientation Estimation}

Several disadvantages with Kalman filter have been reported in the literature [7], [8], therefore we utilized an algorithm that has been shown to provide effective performance at low computational expense and at small sampling rates. Using such a technique, it is feasible to obtain orientation of the sensor in near real-time. This algorithm employs a quaternion representation of orientation [9]. The estimated orientation rate is defined in the following equations:

$$
\left\{\begin{array}{l}
{ }_{E}^{S} q_{t}={ }_{E}^{S} q_{t-1}+{ }_{E}^{S} \dot{q}_{t} \Delta t \\
{ }_{E}^{S} \dot{q}_{t}={ }_{E}^{S} \dot{q}_{\omega, t}-\beta \frac{\nabla f}{\|\nabla f\|}
\end{array}\right.
$$

where

$$
\begin{aligned}
& \nabla f\left({ }_{E}^{S} q, E_{g}, S_{a}\right)=J^{T}\left({ }_{E}^{S} q, E_{g}\right) f\left({ }_{E}^{S} q, E_{g}, S_{a}\right) \\
& S_{a}=\left[0, a_{x}, a_{y}, a_{z}\right] \\
& E_{g}=[0,0,0,1]
\end{aligned}
$$

In this formulation, ${ }_{E}^{S} q_{t}$ and ${ }_{E}^{S} q_{t-1}$ are the orientation of the earth frame relative to the sensor frame at time $t$ and $t-1$ respectively. ${ }_{E}^{S} \dot{q}_{\omega, t}$ is the rate of change of orientation measured by the gyroscopes. $S_{a}$ is the acceleration in the $x, y$ and $z$ axes of the sensor frame, termed $a_{x}, a_{y}, a_{z}$ respectively. The algorithm calculates the orientation ${ }_{E}^{S} q_{t}$ by integrating the estimated rate of change of orientation measured by the gyroscope. Then gyroscope measurement error, $\beta$, was removed in a direction based on accelerometer measurements. We chose the value of $\beta$ to be high enough to minimize errors associated with integral drift but also low enough to avoid noise due to large steps of gradient descent iterations. This gradient descent optimization technique measures only one solution for the sensor orientation by knowing the direction of the gravity in the earth frame. $f$ is the objective function and $J$ is its Jacobean and they are defined by the following equations:

$$
\begin{gathered}
f\left(q, S_{a}\right)=\left[\begin{array}{c}
2\left(q_{2} q_{4}-q_{1} q_{3}\right)-a_{x} \\
2\left(q_{1} q_{2}+q_{3} q_{4}\right)-a_{y} \\
2\left(0.5-q_{2}^{2}-q_{3}^{2}\right)-a_{z}
\end{array}\right] \\
J(q)=\left[\begin{array}{cccc}
-2 q_{3} & 2 q_{4} & -2 q_{1} & 2 q_{2} \\
2 q_{2} & 2 q_{1} & 2 q_{4} & 2 q_{3} \\
0 & -4 q_{2} & -4 q_{3} & 0
\end{array}\right]
\end{gathered}
$$

It is common to quantify orientation sensor performance as the static and dynamic RMS (Root-Mean-Square) errors [6]. The static and dynamic RMS errors for the described orientation estimation algorithm are $<0.8^{\circ}$ and $<1.7^{\circ}$ respectively and hence the algorithm achieves levels of accuracy matching that of the Kalman based algorithm [9].

\section{Position Estimation}

The procedure to determine the position of a swinging instrument (e.g. golf club) is illustrated in the Fig.1. Although in this paper we apply a technique to obtain the $3 \mathrm{D}$ position of a gold club, similar technique can be utilized to measure the position of other sports instruments (e.g. forehand/backhand strokes in tennis). As it is shown in Fig.1, the developed method to calculate the position of the golf club is based on the double integration of the acceleration measurements. It is well-known that position values calculated using this method are only valid for a very short period of time due to the accelerometers' drift error. This means that accumulated error over time during the first and second integration is significant which can result in a remarkable difference between the actual and estimated position. To overcome such a problem and avoid this accumulated error, we detect the back swing and forward swing phases automatically during the full swing action. Therefore, the error produced when velocity and position estimation are calculated for the back swing does not accumulate and influence the velocity and position calculation for the next phase of the swing (i.e forward swing). The motion starts from an initial position and continues until the golf club is lifted to its highest point behind the player and come to rest (back swing). At the end of the back swing phase, linear velocity of the club is close to zero. At this phase, linear velocities will be corrected and thus the error will not be accumulated for the next step, i.e forward swing. The club will then be brought back down to hit the ball and reaches its stopping point in front of the player. 
In order to detect these two phases during the entire swing, total acceleration of the golf club was calculated using the following equation.

$$
a_{\text {total }_{i}}=\sqrt{a_{x_{i}}^{2}+a_{y_{i}}^{2}+a_{z_{i}}^{2}}
$$

At the end of each swing phase, the total acceleration/deceleration is expected to be very small. In fact, although the total acceleration/deceleration is not zero, it should be lower than a certain threshold (obtained via expeimental observation). If it is true, it is considered that the golf club is reaching the end of one phase (e.g back swing) prior to starting the next phase (e.g forward swing). The end of each phase when the total acceleration is below a certain threshold value is defined as rest period (Fig.3). The threshold value used in this paper was $0.18 \mathrm{~g}$. It is necessary to filter the signals in order to eliminate small fluctuations. Butterworth filters are one of the most commonly used digital filters in motion analysis. We opted for digital Butterworth filters as they are fast, simple to use and characterized by a magnitude response that is maximally flat in the passband and monotonic overall. These are all required features to ensure a system generates accurate results in near real-time. We utilized a first order highpass digital Butterworth filter (cutoff frequency $=0.001 \mathrm{~Hz}$ ) and then a first order low-pass digital Butterworth filter (cutoff frequency $=5 \mathrm{~Hz}$ ) to remove noise and hence to accurately identify the rest periods throughout the entire swing. Once the filtered signal is obtained, back swing and forward swing are determined (via rest periods) and therefore the integration and correction of the acceleration measurements can be carried out. Total acceleration signal along with the filtered signal and rest periods are depicted in Fig.3.

In order to compute translational accelerations, it is necessary to rotate local sensor frame acceleration to the global earth frame coordinates using the following equation:

$$
E_{a}={ }_{E}^{S} q \otimes S_{a} \otimes{ }_{E}^{S} q^{*}
$$

In this notation, * denotes conjugate and $E_{a}$ and $S_{a}$ are the acceleration vectors in earth frame and sensor frame, respectively. In order to compute translational velocities, the calculated acceleration in global coordinates are integrated and the velocity at rest periods are set to be equal to zero to correct for the drift error (Fig.4). We then integrate the calculated driftfree velocity to yield position.

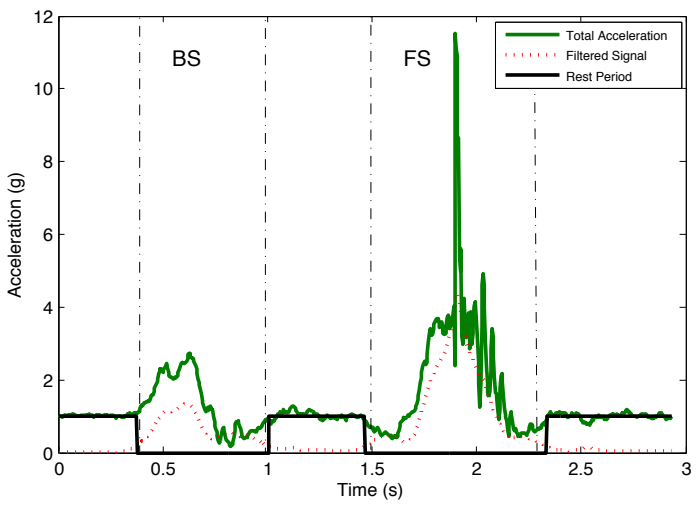

Fig. 3. Total acceleration and filtered signals along with the rest periods are illustrated. BS and FS represent back swing and forward swing, respectively.
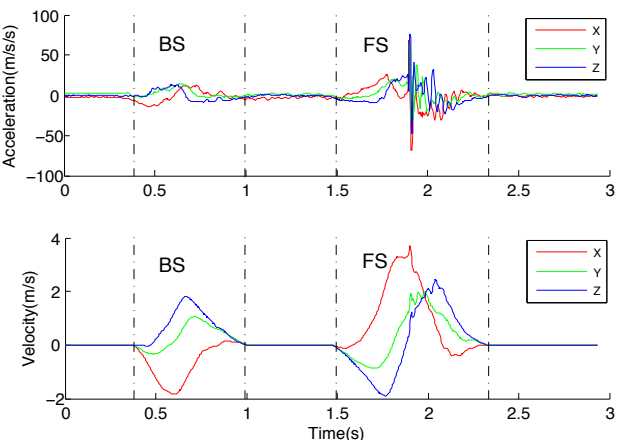

Fig. 4. Raw accelerometer signals (top) along with the corrected (drift-free) velocity (bottom) during the back swing (BS) and forward swing (FS).

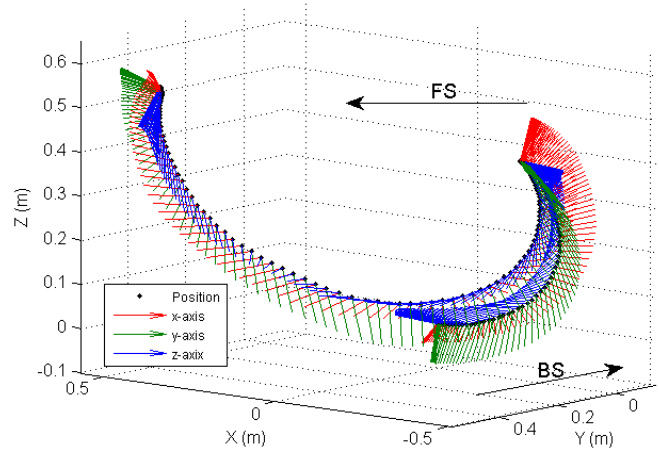

Fig. 5. 3D trajectory (orientation and position) of the sensor attached on the golf club during the back swing (BS) and forward swing (FS) is depicted.

$$
p_{t}=p_{t-1}+v_{t} \times \frac{1}{f},
$$

where $p_{t}$ is position at time $t, p_{t-1}$ is calculated position at time $t-1, v_{t}$ is drift-free velocity at time $t$ and $f$ is the frequency sampling. Calculated orientation as well the position of the golf club during the back swing and forward swing is shown in Fig.5.

\section{Method Evaluation}

The VICON coordinate system is different from the inertial sensor coordinate system. Therefore, it is required to overcome this problem prior to comparing the computed and measured position curves. Although it can easily be achieved through initialization and calibration procedure to obtain the same coordinate system for the two different modalities, in this paper, we propose another technique to resolve the issue if such calibration among modalities maybe not have been performed. The technique is based on applying a rigid registration algorithm on the computed golf club position curve toward the measured curve. In order to do this, we first consider the center of the capturing volume to be $O:\{0,0,0\}$. this is the position of the first sample taken by the VICON system as well as the first sample generated by our devised algorithm, (see Fig. 6). We opted for utilizing the iterative closest point (ICP) algorithm due to its efficiency and reliability [10] to register our computed position curve to that from VICON. We used the $P C L \mathrm{C}++$ library $^{1}$. In addition to the registration step, we also added quality and convergence speed to the registration

\footnotetext{
${ }^{1}$ www.pointclouds.org
} 


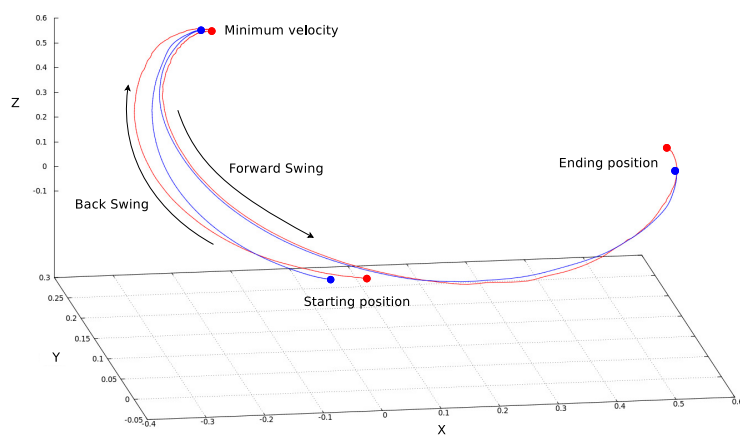

Fig. 6. Trajectory comparison between inertial sensor (red line) and VICON (blue line) is shown.

TABLE II. RMSE AND CROSS CORRELATION RESULTS BETWEEN THE CALCULATED (INERTIAL SENSORS) AND MEASURED (VICON) CURVES.

\begin{tabular}{|c|c|c|}
\hline Trial number & RMS Position $(\mathrm{m})$ & Cross Correlation \\
\hline 1 & 0.1505 & 0.9747 \\
\hline 2 & 0.1257 & 0.9878 \\
\hline 3 & 0.1204 & 0.9884 \\
\hline 4 & 0.1382 & 0.9842 \\
\hline 5 & 0.1226 & 0.9986 \\
\hline 6 & 0.1121 & 0.9975 \\
\hline Mean (SD) & $\mathbf{0 . 1 3 8 2}(\mathbf{0 . 0 3 0 6})$ & $\mathbf{0 . 9 8 8 5}(\mathbf{0 . 0 1 9 8})$ \\
\hline
\end{tabular}

algorithm using principal component analysis (PCA). This geometrical rigid transformation is performed by a momentum analysis, computing the three eigenvectors $\left\{V_{1}, V_{2}, V_{3}\right\}$ of the two point set covariance matrices. We then use a quaternionic rotation to transform our computed point set from the inertial sensor to that from the VICON system.

Let us express this rotation by two quaternions describing two separate rotations, each one defined by an axis and an angle:

$$
\left\{\begin{array}{l}
q_{1}:\left\{V_{1}^{V} \times V_{1}^{I}\right\} \in \mathbb{R}^{3}, V_{1}^{V} \cdot V_{1}^{I} \in \mathbb{R} \\
q_{2}:\left\{V_{2}^{V} \times V_{2}^{I}\right\} \in \mathbb{R}^{3}, V_{2}^{V} \cdot V_{2}^{I} \in \mathbb{R}
\end{array},\right.
$$

where $\times$ is the vector cross product, $V^{V}$ and $V^{I}$ are the set of normalized eigenvectors of the covariance matrices of the VICON system and inertial sensor, respectively. The concatenation of these two rotations $\left(q_{1} \oplus q_{2}\right)$ implies a third rotation described by another quaternion $q$. From the latter, it is feasible to compute an unique rotation matrix to be applied to each point of our computed set points to obtain a new set of points. This set of point is used as an input to the ICP algorithm to register our two trajectories as shown in Fig. 6. This two step registration algorithm is very fast and takes about $6-8 m s$ on our standard desktop machine.

The RMS errors between the calculated and measured results are summarized in Table II. As can be seen, the average RMS errors between the proposed method and the VICON for the golf club position is mean $(S D)=0.1382(0.030) \mathrm{m}$. Also, from the cross correlation results, it is evident that there is a strong agreement between the calculated and measured results $(r=0.9885, p<0.0001)$. This indicates that the measured and calculated results follow the same trend.

\section{CONCLUSiON}

In this paper, we first employed a customized gradient descent optimization algorithm that is capable of computing an error based on an analytically derived Jacobean resulting in a significant reduction in the computation load. This technique was developed to estimate the sensor orientation with respect to the earth frame during the entire swing. In addition, we proposed a signal processing technique to distinguish between a back swing and forward swing phases to obtain drift-free linear velocity from accelerometer signals. This enabled us to measure accurate position of the golf club during the full swing action. The results obtained from the proposed method were compared against the gold standard VICON system and it was shown that there is a close agreement between the calculated and measured results. Due to the low computational load and ability to operate at small sampling rates, the proposed method can be used as a lightweight, inexpensive system to monitor a swing in near real-time in real environment (e.g tennis court, golf course) for extended periods of time.

\section{ACKNOWLEDGMENT}

The research leading to these results has received funding from the European Community's Seventh Framework Programmes (FP7/2013-2016) under grant agreement no. ICT2011-8.2.601170 (REPLAY project) and (FP7/2007-2013) under grant agreement no. ICT-2011-7-287723 (REVERIE project).

\section{REFERENCES}

[1] M. Gowing, A. Ahmadi, F. Destelle, D. S. Monaghan, N. E. OConnor, and K. Moran, "Kinect vs. low-cost inertial sensing for gesture recognition," in MultiMedia Modeling. Springer, 2014, pp. 484-495.

[2] A. Ahmadi, E. Mitchell, F. Destelle, M. Gowing, C. Richter, N. E. O'Connor, and K. Moran, "Automatic activity classification and movement assessment during a sports training session using wearable inertial sensors," in Body Sensor Network, 2014.

[3] A. Avci, S. Bosch, M. Marin-Perianu, R. Marin-Perianu, and P. Havinga, "Activity recognition using inertial sensing for healthcare, wellbeing and sports applications: A survey," in Architecture of computing systems (ARCS), 2010 23rd international conference on. VDE, 2010, pp. 1-10.

[4] H. Ghasemzadeh, V. Loseu, E. Guenterberg, and R. Jafari, "Sport training using body sensor networks: A statistical approach to measure wrist rotation for golf swing," in Proceedings of the Fourth International Conference on Body Area Networks. ICST (Institute for Computer Sciences, Social-Informatics and Telecommunications Engineering), 2009, p. 2.

[5] A. Ahmadi, D. Rowlands, and D. A. James, "Towards a wearable device for skill assessment and skill acquisition of a tennis player during the first serve," Sports Technology, vol. 2, no. 3-4, pp. 129-136, 2009.

[6] A. M. Sabatini, "Quaternion-based extended kalman filter for determining orientation by inertial and magnetic sensing," Biomedical Engineering, IEEE Transactions on, vol. 53, no. 7, pp. 1346-1356, 2006.

[7] J. L. Marins, X. Yun, E. R. Bachmann, R. B. McGhee, and M. J. Zyda, "An extended kalman filter for quaternion-based orientation estimation using marg sensors," in Intelligent Robots and Systems, 2001. Proceedings. 2001 IEEE/RSJ International Conference on, vol. 4. IEEE, 2001, pp. 2003-2011.

[8] H. J. Luinge and P. H. Veltink, "Measuring orientation of human body segments using miniature gyroscopes and accelerometers," Medical and Biological Engineering and computing, vol. 43, no. 2, pp. 273-282, 2005.

[9] S. O. Madgwick, A. J. Harrison, and R. Vaidyanathan, "Estimation of imu and marg orientation using a gradient descent algorithm," in Rehabilitation Robotics (ICORR), 2011 IEEE International Conference on. IEEE, 2011, pp. 1-7.

[10] S. Rusinkiewicz and M. Levoy, "Efficient variants of the ICP algorithm," in Third International Conference on 3D Digital Imaging and Modeling (3DIM), june 2001. 